\title{
Numerical Taxonomy of Selected Slowly Growing Mycobacteria
}

\author{
By G. P. KUBICA \\ Trudeau Institute, Inc., P.O. Box 59, Saranac Lake, New York, 12983 \\ VELLA A. SILCOX \\ Laboratory Branch, Center for Disease Control, Atlanta, Georgia, 30333 \\ AND E. HALL \\ Department of Statistics and Biometry, Emory University, \\ Atlanta, Georgia, 30322, U.S.A.
}

(Received 30 June 1972; revised 29 August 1972)

\begin{abstract}
SUMMARY
A total of 190 strains of slowly growing scotochromogenic and non-photochromogenic mycobacteria, randomly selected from a larger group of $\mathrm{I} 498$ strains, has been analysed using a weighted pair-group method. Dendrograms were constructed from the correlation coefficient data. Mycobacterium flavescens was clearly distinguished from all other scotochromogens. Although not precisely defined, one cluster of pigmented organisms, currently identified only as Group III B, appears to compromise a taxon intermediate between $M$. scrofulaceum and $M$. gordonae. Among the non-photochromogens $M$. triviale is clearly distinct from the others, while the $M$. avium-M. intracellulare aggregate would appear to represent only host-adapted variants of the taxon $M$. avium. Mycobacterium terrae and $M$. novum appear to be very closely related, and only slightly different from $M$. nonchromogenicum. The possibility that these last three represent distinct taxa is considered.
\end{abstract}

\section{INTRODUCTION}

In the preceding paper (Kubica \& Silcox, 1973) the reactions of nearly 1500 strains of slowly growing mycobacteria in 50 test characters in vitro were compared to those of 17 previously established hypothetical median strain (HMS) patterns. This approach enables large masses of assembled data to be handled with relative ease. From previous reports (Kestle, Abbott \& Kubica, I967; Wayne, 1967) the use of HMS patterns seemed to be one method of circumventing the mycobacterial 'mystique' (Wayne, I964), allowing us to find a way through the taxonomy of the genus Mycobacterium.

Although a number of clearly defined clusters did evolve from the earlier study (Kubica \& Silcox, I973), several of the computer-generated groups bearing specific epithets overlapped considerably (i.e. showed high intertaxon similarities). Notable among these were organisms in Runyon Groups II and III (Wayne, Runyon \& Kubica, I969). Within the Group II scotochromogens, Mycobacterium scrofulaceum, M. gordonae and the Group III B organisms (Lewis, Lasché, Armstrong, \& Dunbar, 1960) showed such high levels of intertaxon similarity that a more critical examination of pairs of strains within this complex might resolve the clusters established on the basis of HMS patterns. We also decided to include representative strains of $M$. flavescens to confirm our earlier observations that this taxon was indeed very unlike the other pigmented slowly growing mycobacteria.

Within the Group III non-photochromogens, two aggregates of species appeared to 
require more critical study. The $M y$ cobacterium avium-M. intracellulare complex was one, while the other contained four taxa commonly regarded as environmental saprophytes: $M$. terrae, $M$. nonchromogenicum, $M$. novum and $M$. triviale.

This report describes our attempts to resolve some of these aggregates of taxa using a conventional $n \times n$ matching matrix of randomly selected strains from these larger assemblages.

\section{METHODS}

A total of 190 strains of slowly growing mycobacteria was selected from among those which, in a previous study (Kubica \& Silcox, 1973), were demonstrated to have high intertaxon similarities. These I90 strains were divided into four separate aggregates.

Aggregate I was selected, with an intended bias, from among the Runyon Group II scotochromogens (Wayne et al. 1969). If the III B organisms (Lewis et al. 1960) are indeed distinct from other scotochromogens, such distinction could best be observed if we selected purposely for our study only those showing high matching (M) scores for their respective HMS patterns and relatively low intertaxon $M$ values. Accordingly we chose: I I strains which showed $\mathrm{M}$ values for Mycobacterium scrofulaceum ranging from 9I to $98 \%$; 4 strains with $\mathrm{M}$ values from 83 to $96 \%$ for $M$. gordonae; eight strains with $\mathrm{M}$ values from $8 \mathrm{I}$ to $86 \%$ for $M$. flavescens (these were the highest $\mathrm{M}$ values available in this cluster); and seven strains showing 85 to $92 \% \mathrm{M}$ values for IIIB.

Aggregate 2 consisted of 50 strains chosen from among 397 scotochromogenic mycobacteria. To give adequate representation to the smaller cluster earlier identified (Kubica \& Silcox, 1973) as Mycobacterium flavescens, we selected ten strains from this taxon ( $\mathrm{M}$ values for HMS of $M$. flavescens ranged from $8 \mathrm{I}$ to $85 \%$ ). The remaining 40 strains were randomly selected, ten each from the following clusters: $M$. scrofulaceum (M values for HMS ranged from 77 to $92 \%$ ); $M$. gordonae (M values for HMS ranged from 83 to $96 \%$ ); III B ( $\mathrm{M}$ values for HMS ranged from 79 to $90 \%$ ); and ten strains which showed tie $\mathrm{M}$ values for two or more taxa ranging from $8 \mathrm{I}$ to $94 \%$.

Aggregate 3 was comprized of 50 strains; 25 strains randomly selected from i 30 cultures showed M values from $8 \mathrm{I}$ to $92 \%$ for the HMS of Mycobacterium avium and 25 strains randomly selected from 255 cultures revealed $M$ values from 85 to $98 \%$ for the HMS of $M$. intracellulare.

Aggregate 4 contained 50 randomly selected strains representative of the four smaller clusters identified as not being causative agents of human disease. Ten strains showed $\mathbf{M}$ values for the HMS of Mycobacterium triviale (Kubica et al. 1970) ranging from 85 to I00 \%; ten strains exhibited a range of 87 to $94 \%$ M values for the HMS of $M$. terrae (Wayne, I966); ten strains showed 85 to $98 \% \mathrm{M}$ values for $M$. nonchromogenicum (Tsukamura, I965); ten had $M$ values for the HMS of $M$. novum (Tsukamura, 1967) from 85 to $98 \%$; and ten strains exhibited ties for at least two taxa ranging from 79 to $94 \%$.

The 50 character states used to examine each aggregate were outlined earlier (Kubica \& Silcox, I973). Both positive and negative matches were recorded for all possible pairs of organisms and correlation coefficients were determined (Sokal \& Sneath, 1963).* The matrix of correlation coefficients was analysed for clusters using a weighted pair-group method by

\footnotetext{
* The correlation coefficient, $r$, for each pair of strains was determined from the formula $(a d-b c)$ / $\sqrt{ }[(a+b)(a+c)(b+c)(c+d)]$, where $a$ is the number of negative $(0,0)$ matches, $b$ is the number of 0 , I mismatches, $c$ is the number of $\mathrm{I}$, o mismatches, $d$ is the number of positive $(\mathrm{I}, \mathrm{I})$ matches.

When used with $\mathrm{I}$, o data, $r$ is often called the phi coefficient. The dendrograms generated in this manner are quite similar to those derived from the more common 'matching' $M$ coefficients (using the above notations): $(a+d) /(a+b+c+d)$.
} 


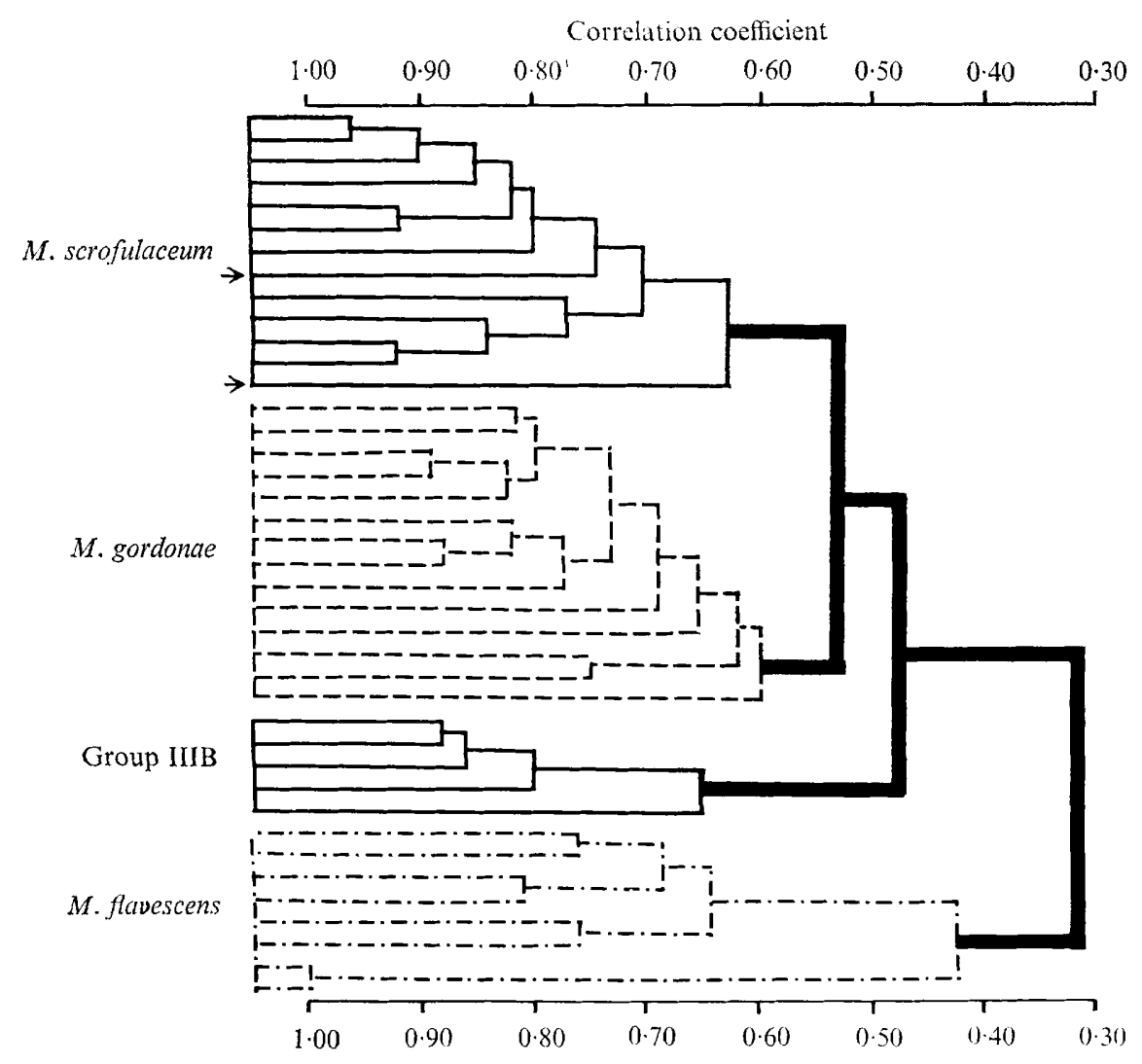

Fig. 1. Correlation coefficient dendrogram of 40 strains of scotochromogenic mycobacteria, selected with intended bias, to strengthen the taxonomic distinctions of Mycobacterium scrofulaceum, $M$. gordonae, M. flavescens and Group IIIB mycobacteria. Arrows indicate two III B strains which clustered with $M$. scrofulaceum. (See text for details.)

which an organism (or group of organisms) is admitted to a cluster based on an average of correlation coefficients of that organism (or group of organisms) with existing members of the cluster (as opposed to single linkage; see Lockhart \& Liston, 1970).

\section{RESULTS}

The correlation coefficient dendrogram for Aggregate I (Fig. I) clearly distinguishes, except for two strains of IIIB organisms which clustered with Mycobacterium scrofulaceum, the four taxa which were selected with admitted bias. Notable here is the distinct separation of Group III B organisms, with a correlation coefficient (CC) of 0.66 , from all other scotochromogenic mycobacteria included in this aggregate. Most of the Mycobacterium flavescens strains clustered together at a $\mathrm{CC}$ of 0.64 , but two strains (last two in $M$. flavescens cluster, Fig. I) showed a CC of $\mathrm{I} \cdot \mathrm{OO}$ for each other, and did not join the rest of the cluster until CC 0.42 was reached. These latter two strains also had lowest $M$ values (8I \%) for the HMS of $M$. flavescens. The clear segregation of $M$. flavescens would support the report of Wayne et al. (1971) that this taxon is markedly different from other more slowly growing scotochromogens. On the other hand, the broad $\mathrm{CC}$ range required ultimately to encompass all 


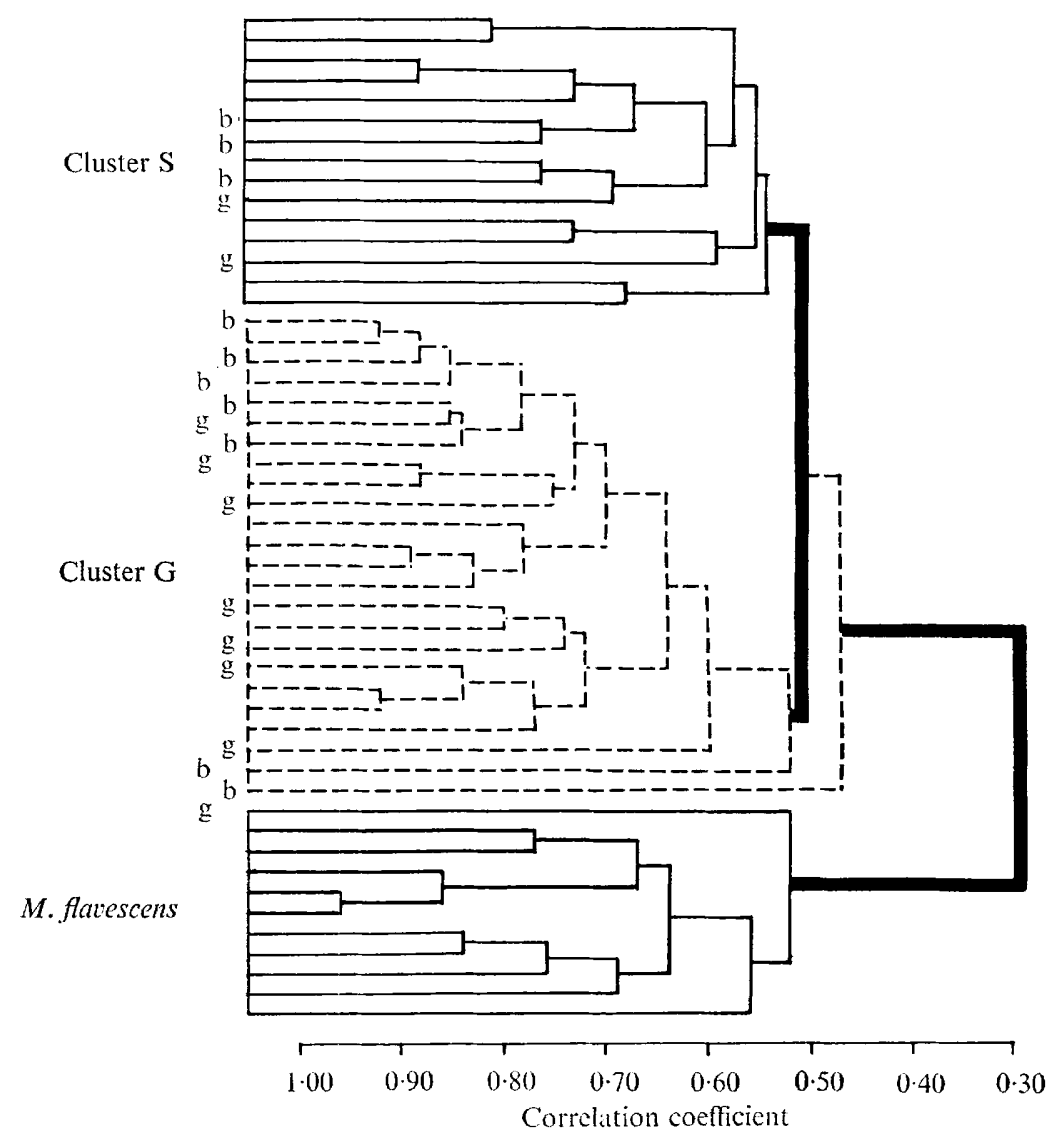

Fig. 2. Correlation coefficient dendrogram of 50 randomly selected scotochromogenic mycobacterial strains. Three major clusters are revealed. $\mathrm{S}=$ Mycobacterium scrofulaceum $; \mathrm{G}=M$. gordonae; and $M$. flavescens. For purposes of identification, the letters $b$ and $g$ refer to strains earlier identified as Group IIIB or $M$. gordonae respectively. (See text for details.)

strains of $M$. flavescens would corroborate the recently reported heterogeneity of this taxon (Jenkins, Marks \& Schaefer, 1972).

In contrast to Aggregate I, the more random selection of scotochromogenic strains results in the segregation of only three clusters: S (predominantly Mycobacterium scrofulaceum), $\mathrm{G}$ (tentatively referred to as $M$. gordonae) and $M$. flavescens (see Fig. 2). Again the dissimilarity of the latter taxon from other scotochromogens is obvious. The apparent absorption of most of the III B organisms into the G (or M. gordonae) cluster was unexpected, although the presence of subclusters within cluster $\mathrm{G}$ would again confirm the recent report of Jenkins et al. (1972) that M. gordonae may be divided by lipid analysis into as many as three distinct groups with their 'provisonal species 3 ' showing some association with human disease. This will be dealt with in Discussion.

Aggregate 3 (Fig. 3) reveals the very close similarity of Mycobacterium avium and $M$. intracellulare in these 50 character states. Although those strains exhibiting highest $M$ values for the HMS patterns of either $M$. avium or M.intracellulare tend to cluster together (clusters $\mathrm{AI}_{\mathrm{I}}$ and $\mathrm{A}$ 2, and cluster B, respectively in Fig. 3), these groups aggregate over a very narrow range of $\mathrm{CC}$ values and occasional intermixing of species is noted. These 


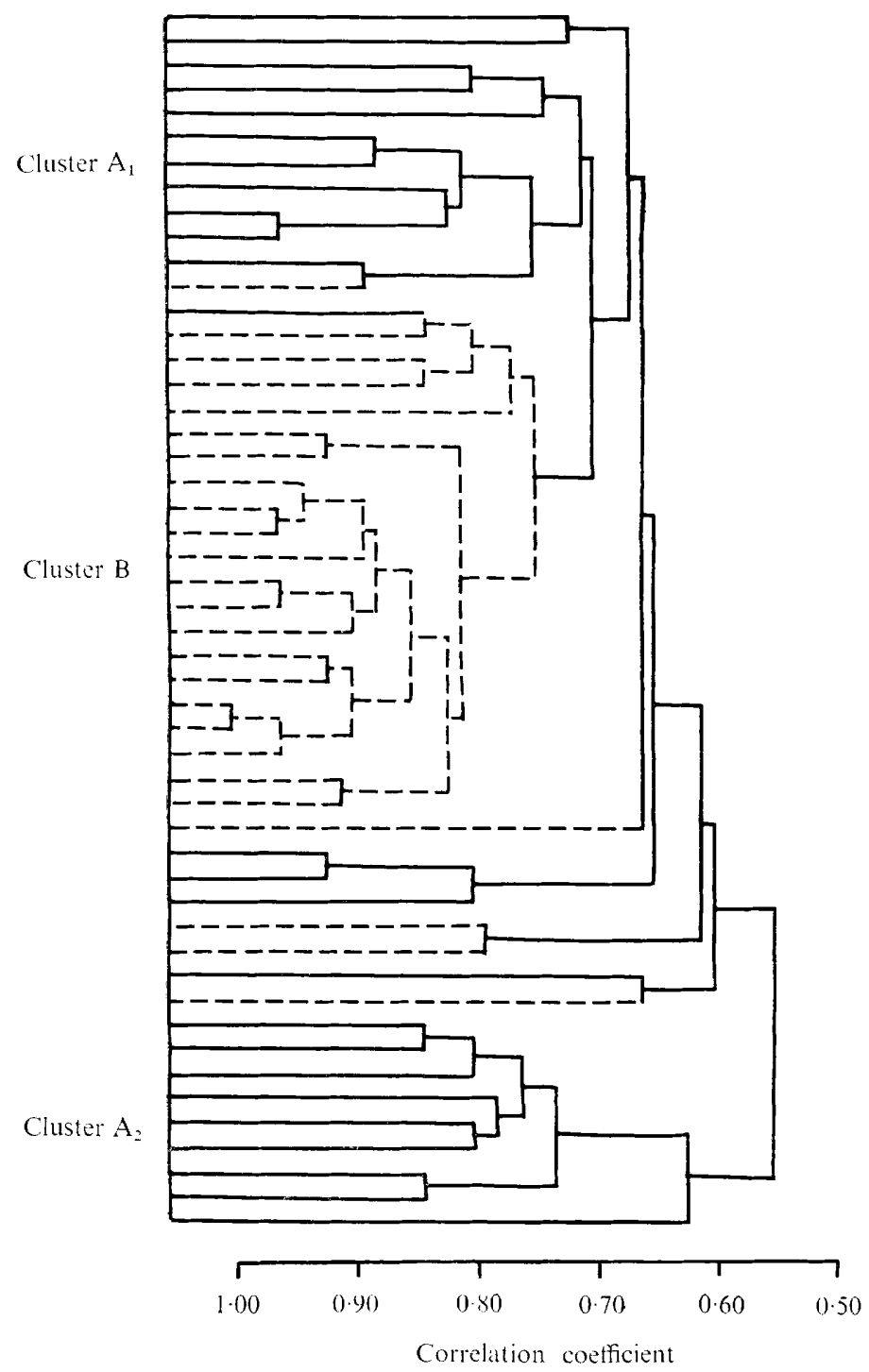

Fig. 3. Correlation coefficient dendrogram of 50 randomly selected strains identified earlier as Mycobacterium avium (solid lines) or $M$. intracellulare (broken lines). Although there was a tendency for segregation of $M$. avium (Clusters $\mathrm{A}_{1}$ and $\mathrm{A}_{2}$ ) from $M$. intracellulare (Cluster $\mathrm{B}$ ), considerable overlap is evident within a narrow $\mathrm{CC}$ range. (See text for details.)

observations support previous reports (Wayne, 1967; Kubica et al. 1970) that these two taxa are indeed very similar, perhaps reflecting host-adapted variants or serotypes of the saine species.

In Aggregate 4 (Fig. 4) the Mycobacterium triviale cluster stands out distinctly from the other strains in the aggregate, joining them at the $0.45 \mathrm{CC}$ line. Strains of $M$. novum and $M$. terrae tended to cluster together, the former cluster containing seven of the ten strains originally designated as $M$. novum, while the latter cluster contained seven of the ten strains originally labelled $M$. terrae. While these two clusters appeared fairly distinct, they fused at 


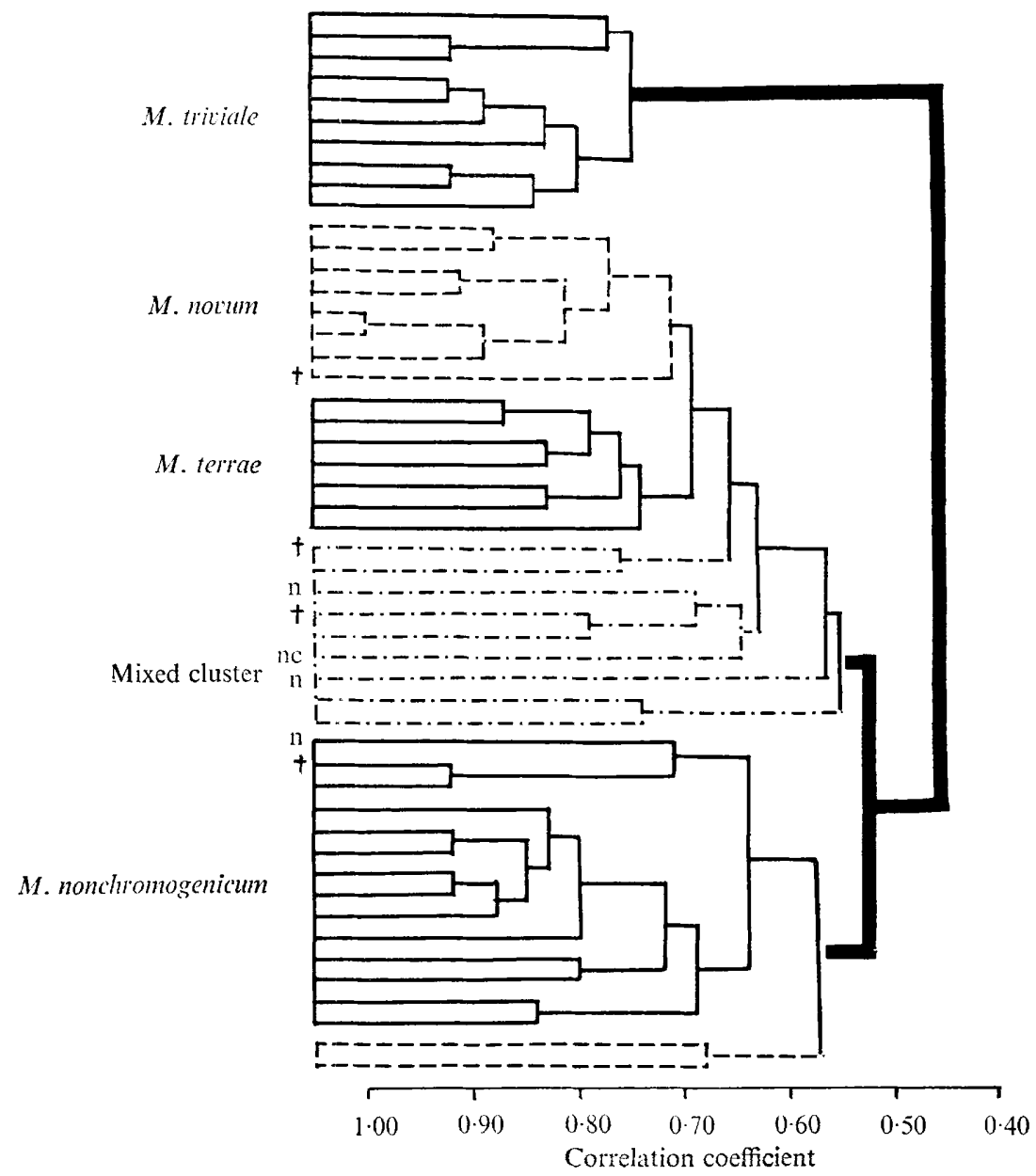

Fig. 4. Correlation coefficient dendrogram of 50 randomly selected non-photochromogenic mycobacteria identified earlier as Mycobacterium triviale, $M$. novum, $M$. terrae and $M$. nonchromogenicum. Although the strains tend to cluster in their respective species, there was some crossing of species boundaries, indicated by lower-case letters: $\mathrm{t}(M$. terrae $), \mathrm{n}(\mathrm{M}$. novm) and nc $(M$. nonchromogenicum). (See text for details.)

the $0.69 \mathrm{CC}$ line, and were subsequently joined by additional strains from the Mixed Cluster (identified earlier by HMS pattern matches as $M$. terrae, $M$. novum, M. nonchromogenicum, or tied values for two or more of these species). Because the members of the Mixed Cluster joined the $M$. novum-M. terrae complex generally only in groups of $\mathrm{I}$ or 2 , this entire cluster probably represents a heterogeneous mixture not clearly definable by the presently employed taxonomic characters. The cluster labelled $M$. nonchromogenicum contained nine of the ten strains originally listed with this epithet, one strain each of $M$. novum and $M$. terrae (see Fig. 4) and five strains which showed tie values for at least two HMS patterns. The intermixing in this dendrogram of strains earlier designated as $M$. terrae, $M$. novum and $M$. nonchromogenicum indicates the close similarity of these three taxa in the test characters here used, and supports earlier observations (Wayne, 1967; Kubica et al. 1970) regarding the close relationship of these three species. 


\section{DISCUSSION}

Mycobacterium flavescens is clearly segregated from the other scotochromogens, supporting the results of a recent co-operative study (Wayne et al. 1971). The heterogeneity of this assemblage, revealed by the broad CC range necessary to encompass even the ten selected strains, confirms the recent report of Jenkins et al. (1972) and strengthens Wayne's (1967) contention that this taxon of intermediate growth rate may be a link between the rapidly and slowly growing mycobacteria.

The III B cluster is more complicated. In a random selection of strains, IIIB tends to cluster with Mycobacterium gordonae (Fig. 2), implying a lack of disease potential (Kestle et al. 1967; Wayne et al. 197r). However, when strain selection was more biased (Fig. I), the IIIB cluster was clearly separable from $M$. scrofulaceum and $M$. gordonae. The early report (Lewis et al. I960) that IIIB could be pathogenic for man, together with recent descriptions of potentially pathogenic unnamed taxa related to $M$. gordonae (see 'Cluster 3 ' of Wayne et al. I97I ; 'provisional species 3' of Jenkins et al. 1972), would support the present data, suggesting that additional work must be done to characterize taxonomically cluster IIIB.

Although the distinction between Mycobacterium scrofulaceum and M. gordonae is not as clear as we would have preferred, the fact that these organisms did segregate into separate clusters confirms the co-operative study report (Wayne et al. 1971) which did distinguish these two taxa. As was mentioned earlier (Kubica \& Silcox, 1973), the reason for the close similarity of these two taxa in this study is the large number of positive and negative matches in a series of tests which do not provide many differential characters for these two species. Additional differential features for $M$. gordonae and $M$. scrofulaceum have been reported (Wayne et al. I97I), while the use of a limited number of highly reliable differential tests (Wayne \& Doubek, I968; Kubica, I973; Pattyn \& Portaels, 1972) makes the separation of these two species a relatively easy matter.

Although there was a general tendency to group together those organisms previously labelled Mycobacterium avium and $M$. intracellulare, the fact that none of the clusters (except possibly A 2; see Fig. 3) was well delineated supports earlier reports (Wayne, I967; Kubica et al. 1970) that these two taxa are closely similar. This strengthens Wayne's view (1967) that the taxa should not be separated. On the other hand, the serological and chemical studies of these taxa (Birn et al. 1967; Schaefer, I968; Marks, Jenkins \& Schaefer, 1970; Yoder \& Schaefer, I97I) indicate clear distinctions of the serotypes within these species suggesting that any genetic changes resulting from host-adapted variations within the $M$. avium- $M$. intracellulare complex may be definitive enough to warrant the taxonomic separation of the two species, providing definitive tests to substantiate their separation are developed. Perhaps results of a forthcoming co-operative study on Runyon Group III nonphotochromogens may shed more light on these two taxa but provisionally we would conclude that all $M$. avium and $M$. intracellulare stains belong together under the epithet $M$. avium, with a number of clearly distinct serotypes.

Among the members of Runyon Group III generally regarded as not being of clinical significance (Wayne et al. 1969), Mycobacterium triviale was clearly distinct from the others, supporting the earlier data obtained in studying HMS patterns (Kubica \& Silcox, 1973). On the other hand, our data tended to equate $M$. novum (Tsukamura, 1967), M. terrae (Wayne, I966) and possibly $M$. nonchromogenicum (Tsukamura, 1965), thus supporting several earlier reports (Wayne, 1967; Kubica et al. 1970). Conversely the data from Tsukamura (1965) taxonomically separates $M$. novum from $M$. nonchromogenicum while 
equating $M$. triviale with the latter (Tsukamura \& Mizuno, I969). As most strains of $M$. novum, $M$. terrae and $M$. nonchromogenicum tended to group together in three separate clusters, even though in a narrow $\mathrm{CC}$ range, the addition of other appropriate tests might strengthen the taxonomic distinction of these three species.

One method of resolving many of the questions posed here, as well as in the many individual reports of others, has been the international co-operative study. Two such studies have been completed (Wayne et al. 1971; Kubica et al. 1972), and four others are in progress. Such studies enable an investigation of many more differential character states than could be conveniently handled by one investigator. The aim, of course, is international recognition and acceptance of a list of epithets in the genus Mycobacterium: this aim is being realized.

\section{REFERENCES}

Birn, K. J., Schaefer, W. B., Jenkins, P. A., Szulga, T. \& Marks, J. (1967). Classification of Mycobacterium avium and related opportunist mycobacteria met in England and Wales. Journal of Higiene 65 , $575^{-589}$.

Jenkins, P. A., Marks, J. \& Schaffer, W. B. (1972). Thin layer chromatography of mycobacterial lipids as an aid to classification: The scotochromogenic mycobacteria, including Mycobacteritum scrofulaceum M. xenopi, M. aquae, M. gordonae, $M$. flavescens. Tubercle 53, I1 $8-127$.

Kestle, D. G., Аввотt, V. D. \& Kubica, G. P. (1967). Differential identification of mycobacteria. II. Subgroups of groups II and III (Runyon) with different clinical significance. American Review of Respiratory Disease 95, 104 I-1052.

KubicA, G. P. (I973). Differential identification of mycobacteria. VII. Key features for identification of clinically significant mycobacteria. American Review of Respiratory Disease (in the press).

Kubica, G. P., Baess, I., Gordon, R. E., Jenkins, P. A., Kwapinski, J. B. G., McDurmont, C., Pattyn, S. R., Saito, H., Silcox, V., Stanford, J. L., Takeya, K. \& Tsukamura, M. (1972). A co-operative numerical analysis of rapidly growing mycobacteria. Journal of General Microbiology 73, 55-70.

KuBICA, G. P. \& SiLcox, V. A. (1973). Numerical taxonomic analysis of some slowly growing mycobacteria using hypothetical median strain patterns. Journal of General Microbiology 74, 149-I 58.

Kubica, G. P., Silcox, V. A., Kilburn, J. O., Smithwick, R. W., Beam, R. E., Jones, W. D., Jun. \& StOTtMeiER, K. D. (1970). Differential identification of mycobacteria. VI. Mycohacterium triviale Kubica sp.nov. International Journal of Systematic Bacteriology 20, I6I-174.

Lewis, A. G., Lasché, E. M., Armstrong, A. L. \& Dunbar, F. P. (I960). A clinical study of the chronic lung disease due to nonphotochromogenic acid-fast bacilli. Annals of Internal Medicine 53, 273-285.

LockhaRT, W. R. \& Liston, J. 1970. Methods of Numerical Taxonomy. Bethesda, Maryland: American Society for Microbiology.

Marks, J., Jenkins, P. A. \& Schaefer, W. B. (1970). Identification and incidence of a third type of Mycobacterium avium. Tubercle 50, 394-395.

Pattyn, S. R. \& Portaels, F. (1972). Identification and clinical significance of mycobacteria. Zentralblatt für Bakteriologie und Hygiene. (Abteilung, I, Originale) A219, I14-140.

SCHAEFER, W. B. (1968). Incidence of the serotypes of Mycobacterium avium and atypical mycobacteria in human and animal disease. American Review of Respiratory Disease 97, 18-23.

Sokal, R. R. \& SNeath, P. H. A. (1963). Principles of Numerical Taxonomy. San Francisco: W. H. Freeman.

TsuKamura, M. (1965). A group of mycobacteria from soil sources resembling nonphotochromogens (Group 3). (In Japanese.) Medicine and Biology 71, I Io-II 3.

TsuKamura, M. (1967). Two types of slowly growing, nonphotochromogenic mycobacteria obtained from soil by the mouse passage method: Mycobacterium terrae and Mycobacterium novum. Japanese Journal of Microbiology II, 163-172.

Tsukamura, M. \& Mizuno, S. (I969). Taxonomy of subgroup ' $V$ ' of the group III nonphotochromogenic mycobacteria. (In Japanese). Kekkaku 44, 13-17.

WaYNe, L. G. (1964). The mycobacterial mystique: deterrent to taxonomy. American Review of Respiratory Disease 9o, 255-257.

WAYNE, L. G. (I966). Classification and identification of mycobacteria. III. Species within group III. American Review of Respiratory Disease 93, 919-928. 
WAYNE, L. G. (1967). Selection of characters for an Adansonian analysis of mycobacterial taxonomy. Journal of Bacteriology 93, I $382-139 \mathrm{I}$.

Wayne, L. G., Dietz, T. M., Gernez-Rieux, C., Jenkins, P. A., Käppler, W., Kubica, G. P., Kwapinski, J. B. G., Meissner, G., Pattyn, S. R., Runyon, E. H., Schröder, K. H., Silcox, V. A., Tacquet, A., Tsukamura, M. \& Wolinsky, E. (197I). A co-operative numerical analysis of scotochromogenic slowly growing mycobacteria. Journal of General Microbiology 66, 255-27 I .

WAYNe, L. G. \& Doubex, J. R. (I968). Diagnostic key to mycobacteria encountered in clinical laboratories. Applied Microbiology 16, 925-831.

Wayne, L. G., Runyon, E. H. \& KubicA, G. P. (1969). Mycobacteria: a guide to nomenclatural usage. American Review of Respiratory Disease 100, 732-734.

Yoder, W. D. \& SChaEfer, W. B. (197I). Comparison of the seroagglutination test with the pathogenicity test in the chicken for the identification of Mycobacterium avium and Mycobacterium intracellulare. American Review of Respiratory Disease 103, 173-178. 